\title{
Application of HPV DNA Testing in Follow-up after Loop Electrosurgical Excision Procedures in Northern Thailand
}

\author{
Surapan Khunamornpong1*, Jongkolnee Settakorn', Kornkanok Sukpan¹, \\ Chumnan Kietpeerakool $^{2}$, Charuwan Tantipalakorn ${ }^{3}$, Prapaporn Suprasert ${ }^{3}$, \\ Sumalee Siriaunkgul ${ }^{1}$
}

\begin{abstract}
Background: HPV DNA testing has been recently introduced as an adjunct test to cytology in the follow-up of patients after treatment for cervical lesions using the loop electrosurgical excision procedure (LEEP). The aim of this study was to evaluate the role of HPV testing in the detection of persistent or recurrent disease after LEEP in patients with cervical epithelial lesions in northern Thailand. Materials and Methods: Patients who underwent LEEP as a treatment for histological low-grade (LSIL) or high-grade squamous intraepithelial lesion (HSIL) or worse at Chiang Mai University Hospital between June 2010 and May 2012 were included. Follow-ups were scheduled at 6-month intervals and continued for 2 years using co-testing (liquid-based cytology and Hybrid Capture 2 [HC2]) at 6 months and 24 months and liquid-based cytology alone at 12 and 18 months. Results: Of 98 patients included, the histological diagnoses for LEEP included LSIL in 16 patients, and HSIL or worse in 82 patients. The LEEP margin status was negative in 84 patients $(85.7 \%)$. At follow-up, 10 patients $(10.2 \%)$ had persistent/recurrent lesions; 4 among LSIL patients $(25.0 \%)$ and 6 in the group with HSIL or worse $(7.3 \%)$. Only 2 of 82 patients $(2.4 \%)$ with HSIL or worse diagnoses had histological HSIL in the persistent/recurrent lesions. Using histologically confirmed LSIL as the threshold for the detection of persistent/recurrent disease, cytology had a higher sensitivity than HC2 $(90.0 \%$ versus $70.0 \%)$. At the 6-month follow-up appointment, combined cytology and HC2 (co-testing) had a higher sensitivity in predicting persistent/recurrent disease $(\mathbf{8 0 . 0 \%})$ compared with that of cytology alone $(\mathbf{7 0 . 0} \%)$ and HC2 $(50.0 \%)$. Conclusions: After LEEP with a negative surgical margin, the rate of persistent/recurrent lesions is low. The addition of HPV testing at the 6-month visit to the usual cytology schedule may be an effective approach in the follow-up after LEEP.
\end{abstract}

Keywords: Cervix - squamous intraepithelial lesion - LEEP - follow-up - HPV test - Thailand

Asian Pac J Cancer Prev, 16 (14), 6093-6097

\section{Introduction}

Human papillomavirus (HPV) is a necessary cause of almost all cervical cancers and precancerous lesions. Cervical cancer screening using cytology and/or highrisk HPV DNA testing helps detect precancerous lesions or early cervical cancers that can be surgically treated (Siriaunkgul et al., 2014). Cervical conization with the loop electrosurgical excision procedure (LEEP) has an important role in both diagnosis and treatment of cervical epithelial lesions by the removal of them for a thorough pathologic examination (Wright et al., 2003; Sangkarat et al., 2014).

In patients who undergo conization as a conservative treatment for cervical epithelial lesions, a follow-up evaluation is important in order to identify the presence of persistent or recurrent disease. Cytology ( \pm colposcopy) is the standard method used in the follow-up after conization (Wright et al., 2003). Recently, HPV testing has been introduced as an additional test in combination with cytology in the patients' follow-up, and many studies on this topic have been published (Nobbenhuis et al., 2001; Paraskevaidis et al., 2004). In previous studies, posttreatment HPV testing has been reported to have a high sensitivity and negative predictive value in the prediction of persistent/recurrent lesions, which suggests a possibility that HPV testing may help reduce the number of follow-up visits (Houfflin Debarge et al., 2003; Alonso et al., 2006; Kitchener et al., 2008; Jeong et al., 2009; Lubrano et al., 2012; Ryu et al., 2012). However, there is very limited information regarding the application of HPV testing in patient follow-up after LEEP in the populations of Southeast Asia or developing countries, where cervical cancer incidence is high (Nessa et al., 2014).

${ }^{1}$ Department of Pathology, Faculty of Medicine, Chiang Mai University, Chiang Mai, ${ }^{2}$ Department of Obstetrics and Gynecology, Faculty of Medicine, Khon Kaen University, Khon Kaen, ${ }^{3}$ Department of Obstetrics and Gynecology, Faculty of Medicine, Chiang Mai University, Chiang Mai, Thailand*For correspondence: skhunamo@yahoo.com 
The aim of this study is to evaluate the role of highrisk HPV DNA testing in the detection of persistent or recurrent disease after LEEP in patients with cervical epithelial lesions in northern Thailand.

\section{Materials and Methods}

The study was approved by Institutional Ethics Committee. Patients who underwent LEEP at Chiang Mai University Hospital between June 2010 and May 2012 were invited onto the study. Informed consent was obtained. In this institution, LEEP was carried out in all women with cytology results or cervical biopsy histology of high-grade cervical epithelial lesions. For women with minor cervical cytologic abnormalities, LEEP was performed if the initial work-up results suggested the presence of high-grade lesions. However, LEEP following colposcopy without intervening histological diagnosis may be performed in women with minor cytologic abnormalities, whose colposcopic findings were suggestive of high-grade disease. In most patients, endocervical curettage was also performed after LEEP. In patients with a positive surgical margin for high-grade epithelial lesions, a repeat LEEP (re-LEEP) was justified when feasible. The histological diagnosis in each patient was based on the most severe lesion detected in either the LEEP/re-LEEP specimen or the cervical biopsy prior to LEEP. In patients who underwent a re-LEEP, the margin status was determined in the re-LEEP specimens.

Inclusion criteria were patients with a histological diagnosis of squamous intraepithelial lesion (SIL), either low-grade (LSIL) or high-grade (HSIL), or microinvasive carcinoma who underwent LEEP conization as a treatment with the aim of achieving a complete removal of the epithelial lesions. Exclusion criteria were patients who had no histological confirmation of SIL in LEEP or prior cervical biopsy, patients who underwent LEEP as a diagnostic test prior to hysterectomy, or patients with incomplete follow-up and with no persistent/recurrent lesion detected.

Follow-ups were scheduled at 6-month intervals during the 2 years after $\operatorname{LEEP}(6,12,18$, and 24 months), using co-testing (HPV test and liquid-based cytology) at 6 month and 24 months and cytology alone at 12 and 18 months. Patients who had negative test results in all follow-up visits during the 2-year period returned to the annual screening program. Patients who had cytological abnormalities or HPV positivity at 24 months received a colposcopic evaluation. During the colposcopy, a cervical biopsy was performed when any abnormality was detected. If the transformation zone was not visible or no abnormality was identified, endocervical sampling by brushing cytology or curettage was obtained. The presence of histologically confirmed epithelial lesions (HSIL or LSIL) within the first 6 months was considered as persistent disease and after 6 months as recurrent disease (Sangkarat et al., 2014).

In each follow-up visit, a cervical specimen was collected using a plastic spatula and a cytobrush. The cervical samples were transferred from the collecting devices into PreservCyt solution (Cytyc Corporation,
Boxborough, MA, USA) for liquid-based cytology preparation (ThinPrep: Hologic, Marlborough, MA, USA) and for HPV testing (Hybrid Capture 2 [HC2]: Qiagen, Hilden, Germany). According to the manufacturer's information, HC2 is designed to detect 13 high-risk HPV genotypes $(16,18,31,33,35,39,45,51,52,56,58,59$ and 68). Cervical cytology was reported according to the 2001 Bethesda System. Cytology was considered as abnormal when the result was at least atypical squamous cells of undetermined significance (ASC-US). A positive HC2 test is defined with a quantitative threshold from a relative light unit/positive control (RLU/PC) ratio $\geq 1.0$.

The data including patient age, LEEP histological diagnosis, LEEP margin status, endocervical curettage results at LEEP, and the results of HC2 and cytology at follow-up were analyzed using STATA version 11 (StataCorp LP, College Station, TX, USA). Comparison of the difference between variables was evaluated using Fisher Exact test. A p value $<0.05$ was considered statistically significant. Accuracy values of the follow-up testing methods in the prediction of persistent/recurrent disease were calculated.

\section{Results}

There were 98 patients included in this study. A further 4 patients were excluded due to incomplete follow-up HPV testing and/or cytology at 24 months. The histological diagnoses included LSIL in 16 patients, HSIL in 80 patients, and microinvasive squamous cell carcinoma in 2 patients (stage IA1: extent of stromal invasion $<1 \mathrm{~mm}$ and without lymphovascular space invasion). The mean patient age was 45.3 \pm SD 9.2 years (45.1 years for LSIL and 45.4 years for HSIL or worse). Immediate re-LEEP was performed in 14 patients. The LEEP margin status was negative in 84 patients $(85.7 \%)$. The remaining 14 patients (14.3\%) had either positive (12 patients) or uncertain/ non-evaluable margin status (2 patients).

At follow-up, 10 patients $(10.2 \%)$ had persistent/ recurrent lesions (1 persistent, 9 recurrent); 6 from 82 patients $(7.3 \%)$ after the diagnosis of high-grade lesions (HSIL or worse) and 4 from 16 patients $(25.0 \%)$ after a diagnosis of LSIL. The persistent/recurrent lesions in 6 patients with HSIL or worse diagnosis were histologically identified as HSIL in 2 patients and LSIL in 4 patients. All 4 patients with persistent/recurrent disease after the LSIL diagnosis had LSIL histology in the recurrent lesions. Three patients who had persistent /recurrent disease underwent a subsequent hysterectomy after 12 months of follow-up. The two patients with stage IA1 squamous cell carcinoma in LEEP had negative follow-up testing (HPV and cytology) in all visits, without any clinical evidence of recurrence.

The rate of persistent/recurrent lesions among the patients with LSIL diagnosis was higher than that of the patients with HSIL or worse, with a marginal significance ( $25.0 \%$ vs $7.3 \%, \mathrm{p}=0.055)$. Thus, data of both groups were separately analyzed for the factors predicting persistent/ recurrent disease; those with HSIL or worse diagnosis (Table 1) and those with LSIL diagnosis (Table 2).

Among patients with HSIL or worse diagnosis, age 
$\leq 35$ years and positive or uncertain margin status were significantly associated with a higher risk for persistent/ recurrent disease ( $\mathrm{p}=0.038$ and $\mathrm{p}=0.029$, respectively). At follow-up, positive HC2 ( $\mathrm{p}=0.038$ ), positive cytology $(\mathrm{p}=0.001)$, and positive combined test results $(\mathrm{p}<0.001)$ were significantly predictive of persistent/recurrent disease. At 6 months after LEEP, cytology and co-testing were also significantly predictive of persistent/recurrent disease $(\mathrm{p}<0.001$, each), but HC2 alone was not ( $\mathrm{p}=0.080)$. In the case of positive surgical margin status, the risk of persistent/recurrent disease was not significantly different between the margin types (endocervical versus ectocervical). Among the patients with LSIL diagnosis, HC2 positivity was the only variable that showed a significant association with persistent/recurrent disease $(\mathrm{p}=0.019)$.

The rate of positive $\mathrm{HC} 2$ was higher among the patients with a positive or uncertain LEEP margin status ( 5 of 14 or $35.7 \%$ ) than those with a negative margin (14 of 84 or $16.7 \%$ ), but the difference was not significant $(\mathrm{p}=0.138)$. One of 2 patients with persistent/recurrent HSIL had the cytology result of HSIL with a negative $\mathrm{HC} 2$ test (RLU/PC ratio of 0.84 ) at the 6-month follow-

Table 1. Comparison of Variables for Persistent/ recurrent Disease in 82 Patients with a Diagnosis of HSIL or Worse

\begin{tabular}{|c|c|c|c|}
\hline Variable & $\begin{array}{c}\text { Total } \\
\text { No. }(\%)\end{array}$ & $\begin{array}{l}\text { No. of } \\
\text { patients with } \\
\text { persistent/ } \\
\text { recurrent } \\
\text { disease }\end{array}$ & $\begin{array}{l}\text { No. of } \\
\text { atients with } \\
\text { persistent/ } \\
\text { recurrent } \\
\text { high-grade } \\
\text { lesions }\end{array}$ \\
\hline \multicolumn{4}{|l|}{ Age } \\
\hline$\leq 35$ years & $12(14.6)$ & $3(25.0 \%)^{*}$ & $0(0 \%)$ \\
\hline$>35$ years & $70(85.4)$ & $3(4.3 \%)$ & $2(2.9 \%)$ \\
\hline \multicolumn{4}{|l|}{ Margin status } \\
\hline Positive or uncertain & $11(13.4)$ & $3(27.3 \%)^{*}$ & $0(0 \%)$ \\
\hline Negative & $71(86.6)$ & $3(4.2 \%)$ & $2(2.8 \%)$ \\
\hline \multicolumn{4}{|l|}{ Endocervical curettage } \\
\hline Positive & $4(4.9)$ & $1(25.0 \%)$ & $0(0 \%)$ \\
\hline Negative & $72(87.8)$ & $4(5.6 \%)$ & $1(1.4 \%)$ \\
\hline Not available & $6(7.3)$ & $1(16.7 \%)$ & $1(16.7 \%)$ \\
\hline \multicolumn{4}{|l|}{$\mathrm{HC} 2$ at 6 months } \\
\hline Positive & $7(8.5)$ & $2(28.6 \%)$ & $1(14.3 \%)$ \\
\hline Negative & $75(91.5)$ & $4(5.3 \%)$ & $1(1.3 \%)$ \\
\hline \multicolumn{4}{|l|}{ Cytology at 6 months } \\
\hline Positive & $7(8.5)$ & $4(57.1 \%) * * *$ & $2(28.6 \%)$ \\
\hline Negative & $75(91.5)$ & $2(2.7 \%)$ & $0(0 \%)$ \\
\hline \multicolumn{4}{|c|}{ Combined $\mathrm{HC} 2$ and cytology at 6 months } \\
\hline Positive & $16(19.5)$ & $5(31.3 \%) * * *$ & $2(12.5 \%)$ \\
\hline Negative & $66(80.5)$ & $1(1.5 \%)$ & $0(\%)$ \\
\hline \multicolumn{4}{|l|}{ Any positive $\mathrm{HC} 2$} \\
\hline Yes & $12(14.6)$ & $3(25.0 \%)^{*}$ & $1(8.3 \%)$ \\
\hline No & $70(85.4)$ & $3(4.3 \%)$ & $1(4.3 \%)$ \\
\hline \multicolumn{4}{|l|}{ Any positive cytology } \\
\hline Yes & $17(20.7)$ & $5(31.3 \%) * *$ & $2(11.8 \%)$ \\
\hline No & $65(79.3)$ & $1(1.5 \%)$ & $0(0 \%)$ \\
\hline \multicolumn{4}{|c|}{ Combined $\mathrm{HC} 2$ and cytology } \\
\hline Positive (any test) & $23(28.0)$ & $6(26.1 \%) * * *$ & $2(8.7 \%)$ \\
\hline Negative & $59(72.0)$ & $0(0 \%)$ & $0(0 \%)$ \\
\hline Total & $82(100)$ & $6(7.3 \%)$ & $2(2.4 \%)$ \\
\hline
\end{tabular}

DOI:http://dx.doi.org/10.7314/APJCP.2015.16.14.6093

HPV Testing in Follow-up after LEEP in Northern Thailand up appointment. Of 68 patients who were HC2-negative at 6 months and had negative cytology results within 24 months, only one patient $(1.5 \%)$ had recurrent LSIL and this patient was HC2- positive at 24 months. Three patients who were HC2-positive but cytology-negative at 24 months had negative colposcopic findings and biopsy.

The sensitivity, specificity, positive predictive value (PPV), and negative predictive value (NPV) of HC2 and cytology in the prediction of persistent/recurrent lesions

Table 2. Comparison of Variables for Persistent/ recurrent Disease in 16 Patients with a Diagnosis of LSIL

\begin{tabular}{|c|c|c|}
\hline Variable & Total No. $(\%)$ & $\begin{array}{c}\text { No. of patients with } \\
\text { persistent/recurrent } \\
\text { disease }\end{array}$ \\
\hline \multicolumn{3}{|l|}{ Age } \\
\hline$\leq 35$ years & $1(6.3)$ & $1(100 \%)$ \\
\hline$>35$ years & $15(93.7)$ & $3(20.0 \%)$ \\
\hline \multicolumn{3}{|l|}{ Margin status } \\
\hline Positive or uncertain & $3(18.8)$ & $2(66.7 \%)$ \\
\hline Negative & $13(81.2)$ & $2(15.4 \%)$ \\
\hline \multicolumn{3}{|l|}{ Endocervical curettage } \\
\hline Positive & $0(0)$ & $0(0 \%)$ \\
\hline Negative & $16(100)$ & $4(25.0 \%)$ \\
\hline \multicolumn{3}{|l|}{$\mathrm{HC} 2$ at 6 months } \\
\hline Positive & $6(37.5)$ & $3(50.0 \%)$ \\
\hline Negative & $10(62.5)$ & $1(10.0 \%)$ \\
\hline \multicolumn{3}{|l|}{ Cytology at 6 months } \\
\hline Positive & $5(31.3)$ & $3(60.0 \%)$ \\
\hline Negative & $11(68.7)$ & $1(9.1 \%)$ \\
\hline \multicolumn{3}{|c|}{ Combined HC2 and cytology at 6 months } \\
\hline Positive & $7(43.8)$ & $3(42.9 \%)$ \\
\hline Negative & $9(56.2)$ & $1(11.1 \%)$ \\
\hline \multicolumn{3}{|l|}{ Any positive $\mathrm{HC} 2$} \\
\hline Yes & $7(43.8)$ & $4(42.9 \%)^{*}$ \\
\hline No & $9(56.2)$ & $0(0 \%)$ \\
\hline \multicolumn{3}{|l|}{ Any positive cytology } \\
\hline Yes & $9(56.2)$ & $4(44.4 \%)$ \\
\hline No & $7(43.8)$ & $0(0 \%)$ \\
\hline \multicolumn{3}{|c|}{ Combined $\mathrm{HC} 2$ and cytology } \\
\hline Positive (any test) & $9(56.2)$ & $4(44.4 \%)$ \\
\hline Negative & $7(43.8)$ & $0(0 \%)$ \\
\hline Total & $16(100)$ & $4(25.0 \%)$ \\
\hline
\end{tabular}

Table 3. Performance of the Tests Used in the Detection of Persistent/Recurrent Lesions at LSIL Threshold

Sensitivity Specificity Positive Negative

(\%) $\quad(\%) \quad$ predictive predictive value $(\%)$ value $(\%)$

\begin{tabular}{lcllc}
\hline All patients $(\mathrm{n}=98)$ & & & & \\
HC2 at 6 months & 50 & 90.9 & 38.5 & 94.1 \\
Cytology at 6 months & 70 & 94.3 & 58.3 & 96.5 \\
Co-test at 6 months & 80 & 83 & 34.8 & 97.3 \\
HC2 at 6 and 24 months & 70 & 86.4 & 36.8 & 96.2 \\
Cytology follow-ups & 90 & 80.7 & 34.6 & 98.6 \\
Combined tests & 100 & 75 & 31.3 & 100 \\
Patients with HSIL or worse (n=82) & & & \\
HC2 at 6 months & 33.3 & 93.4 & 28.6 & 94.7 \\
Cytology at 6 months & 66.7 & 96.1 & 57.1 & 97.3 \\
Co-test at 6 months & 83.3 & 85.5 & 31.3 & 98.5 \\
HC2 at 6 and 24 months & 50 & 88.2 & 25 & 95.7 \\
Cytology follow-ups & 83.3 & 84.2 & 29.4 & 98.5 \\
Combined tests & 100 & 77.6 & 26.1 & 100 \\
\hline
\end{tabular}


(using the threshold of LSIL) among all patients and among 82 patients with HSIL or worse diagnosis are presented in Table 3. Cytology had a higher sensitivity than $\mathrm{HC} 2$. At the 6-month follow-up appointments, cotesting had a higher sensitivity and NPV in predicting persistent/recurrent disease compared with that of cytology or $\mathrm{HC} 2$ alone; however, the specificity and PPV of co-testing were lower. Among the patients with HSIL or worse diagnosis, the performance of co-testing at the 6-month follow-up was comparable to that of the followup cytology until 24 months.

Among 16 patients with LSIL diagnosis, the sensitivity and NPV of HPV testing and cytology in predicting persistent/recurrent disease was high (100\%), although HPV testing tended to have a higher specificity $(75.0 \%$ vs $58.3 \%)$ and PPV (57.1\% vs $44.4 \%)$. However, when compared to cytology alone, the combined results of HPV testing and cytology did not improve the detection of persistent/recurrent disease.

\section{Discussion}

Persistence or recurrence of SIL after LEEP conization was reported in 5-18\% of patients (Houfflin Debarge et al., 2003; Jeong et al., 2009; Lubrano et al., 2012; Ryu et al., 2012). Several variables have been proposed to be predictive of persistent/recurrent disease including age, pre-treatment cytology, histological diagnosis in LEEP, surgical margin status, viral load in HPV testing (pre- or post-treatment), and pre-treatment HPV genotypes (Song et al., 2006; Jeong et al., 2009; Nam et al., 2009; Ryu et al., 2012). In many studies, the surgical margin status of LEEP has been reported to be one of the most important predictors of persistent/recurrent disease (Houfflin Debarge et al., 2003; Ryu et al., 2012). The difference in rate of positive LEEP margins (11-46\%) (Houfflin Debarge et al., 2003) may partly contribute to the variation of recurrence rate after LEEP in different studies.

In patients treated for histological HSIL, the recurrence rate was reported to be higher in cases with a positive margin compared to those with a negative margin (27-36\% versus 5-12\%) (Houfflin Debarge et al., 2003; Alonso et al., 2006). The finding in our study $(27.3 \%$ versus $4.2 \%$ ) is in keeping with such reported rates. The rate of persistent/recurrent lesions as HSIL was only $2.4 \%$ in this study which was lower than in an earlier study (6.6\%) (Ryu et al., 2012). The very low number of patients with HSIL who had persistent/recurrent disease in this study limits the statistical analysis for the predictive factors for persistence/recurrence.

The positive rate of HPV testing using HC2 after LEEP has been reported to be approximately $25 \%$ (Lubrano et al., 2012; Ryu et al., 2012). This rate tended to be higher in the cases with positive margin status than those with negative margin status (36-41\% versus $17-31 \%$ ), but the difference was not significant in either the current or previous studies (Houfflin Debarge et al., 2003; Song et al., 2006). The post-treatment HPV-positive rate may also be variable with different HPV detection methods (Lubrano et al., 2012).

In previous studies, HPV testing has been reported to be more sensitive than cytology in the detection of persistent/recurrent lesions after LEEP with a diagnosis of HSIL (Alonso et al., 2006; Ryu et al., 2012). HPV testing at 6-12 months has been found to have a very high sensitivity and NPV (both approaching 100\%) in predicting persistent/recurrent disease (Alonso et al., 2006; Jeong et al., 2009; Lubrano et al., 2012). In the present study, the performance of co-testing at 6-months in the patients with HSIL or worse diagnoses was comparable to that of follow-up cytology until 24 months. However, the sensitivity of HPV testing in this study was lower than that previously reported. The low number of persistent/ recurrent cases in this study limited the assessment of performance difference between different testing methods.

Follow-up after LEEP in patients with a diagnosis of LSIL is important as approximately $12 \%$ of these patients may have a progression of the disease to HSIL (Alonso et al., 2007). In the present and previous studies, persistence/recurrence rate after LEEP tended to be higher in the patients with a diagnosis of LSIL than those patients with HSIL diagnosis (25-33\% versus 7-24\%) (Alonso et al., 2007). This may be partly explained by the nature of SIL as HSIL is a neoplastic lesion of monoclonal origin whereas LSIL is more likely to represent a productive HPV infection (Ueda et al., 2003). Thus, a negative LEEP margin in the excision of HSIL may suggest a high possibility for a complete removal of the lesion. In patients with a diagnosis of LSIL, the sensitivity of HPV testing and cytology in predicting persistence/recurrence was similarly high. However, combining the result of HPV testing with that of the cytology did not improve the probablitiy in predicting persistent/recurrent disease in the patients with LSIL diagnosis, when compared with cytology alone.

Low compliance to follow-up is an important issue in the consideration of management of the patients. A previous study has noted this problem in patients in Thailand (Rattanalappaiboon et al., 2014). This factor supports the treatment policy to remove the entire lesion by achieving a negative conization margin to reduce the risk of persistent/recurrent disease, although the complication rates may be increased with extensive conization (Sangkarat et al., 2014). In the setting of this study where this policy for LEEP is applied, co-testing at 6 months after LEEP and cytology in the other visits may be an effective approach in the follow-up of patients after LEEP.

One of the limitations in this study is the absence of pre-treatment HPV testing for a comparison with posttreatment HPV results. Previous studies have shown a very high rate of positive pre-treatment HPV testing in patients with a diagnosis of HSIL (95-97\%) and also that a high pre-treatment viral load is a risk factor for persistent/recurrent disease (Song et al., 2006; Nam et al., 2009). In the present study, one of 2 patients with persistent/recurrent HSIL was HPV-negative. In a previous study, 3 of 20 (15\%) patients who had persistent/recurrent HSIL had a negative HC2 test (Lubrano et al., 2012). The finding suggests that HPV testing alone may not be an effective follow-up test and co-testing should be preferred. Another limitation in the present study is the 
use of liquid-based cytology in follow-up, and the results may not be directly compared with the practice using conventional Pap cytology. In a recent large scale study, liquid-based cytology was found to increase the detection rate of ASC-US and LSIL in women younger than 40 years compared to conventional cytology; however, there is no significant difference in the detection of HSIL or worse (Sigurdsson, 2013).

In conclusion, the rate of persistent/recurrent disease after LEEP with a negative surgical margin is low, and the rate of persistent/recurrent high-grade lesions is very low. Combining HPV testing with cytology may increase the sensitivity in the detection of persistent/recurrent disease after LEEP conization, compared to cytology alone. The addition of HPV testing at the 6-month visit to the usual cytology schedule may be an effective approach in the follow-up after LEEP.

\section{Acknowledgements}

This study was supported by 1) the National Research University Project, under Thailand's Office of the Higher Education Commission; and 2) the Faculty of Medicine, Chiang Mai University. The authors declare that there is no conflict of interest.

\section{References}

Alonso I, Torne A, Puig-Tintore LM, et al (2006). Pre- and post-conization high-risk HPV testing predicts residual/ recurrent disease in patients treated for CIN 2-3. Gynecol Oncol, 103, 631-6.

Alonso I, Torne A, Puig-Tintore LM, et al (2007). High-risk cervical epithelial neoplasia grade 1 treated by loop electrosurgical excision: follow-up and value of HPV testing. Am J Obstet Gynecol, 197, 359.

Houfflin Debarge V, Collinet P, Vinatier D, et al (2003). Value of human papillomavirus testing after conization by loop electrosurgical excision for high-grade squamous intraepithelial lesions. Gynecol Oncol, 90, 587-92.

Jeong NH, Lee NW, Kim HJ, et al (2009). High-risk human papillomavirus testing for monitoring patients treated for high-grade cervical intraepithelial neoplasia. J Obstet Gynaecol Res, 35, 706-11.

Kitchener HC, Walker PG, Nelson L, et al (2008). HPV testing as an adjunct to cytology in the follow up of women treated for cervical intraepithelial neoplasia. BJOG, 115, 1001-7.

Lubrano A, Medina N, Benito V, et al (2012). Follow-up after LLETZ: a study of 682 cases of CIN 2-CIN 3 in a single institution. Eur J Obstet Gynecol Reprod Biol, 161, 71-4.

Nam K, Chung S, Kim J, et al (2009). Factors associated with HPV persistence after conization in patients with negative margins. J Gynecol Oncol, 20, 91-5.

Nessa A, Rashid MH, Jahan M, et al (2014). Role of the HPV DNA test in follow-up of treated cervical intraepithelial neoplasia in Bangladesh. Asian Pac J Cancer Prev, 15, 8063-7.

Nobbenhuis MA, Meijer CJ, van den Brule AJ, et al (2001). Addition of high-risk HPV testing improves the current guidelines on follow-up after treatment for cervical intraepithelial neoplasia. Br J Cancer, 84, 796-801.

Paraskevaidis E, Arbyn M, Sotiriadis A, et al (2004). The role of HPV DNA testing in the follow-up period after treatment for CIN: a systematic review of the literature. Cancer Treat Rev, 30, 205-11.

Rattanalappaiboon D, Kietpeerakool C, Kleebkaow P, et al (2014). Factors affecting compliance in the first year of postcolposcopy surveillance among women with a high incidence of cervical cancer. Int J Gynaecol Obstet, 124, 160-3.

Ryu A, Nam K, Kwak J, et al (2012). Early human papillomavirus testing predicts residual/recurrent disease after LEEP. $J$ Gynecol Oncol, 23, 217-25.

Sangkarat S, Ruengkhachorn I, Benjapibal M, et al (2014). Longterm outcomes of a loop electrosurgical excision procedure for cervical intraepithelial neoplasia in a high incidence country. Asian Pac J Cancer Prev, 15, 1035-9.

Sigurdsson K (2013). Is a liquid-based cytology more sensitive than a conventional Pap smear? Cytopathology, 24, 254-63.

Siriaunkgul S, Settakorn J, Sukpan K, et al (2014). Populationbased cervical cancer screening using high-risk HPV DNA test and liquid-based cytology in northern Thailand. Asian Pac J Cancer Prev, 15, 6837-42.

Song SH, Lee JK, Oh MJ, et al (2006). Persistent HPV infection after conization in patients with negative margins. Gynecol Oncol, 101, 418-22.

Ueda Y, Enomoto T, Miyatake T, et al (2003). Monoclonal expansion with integration of high-risk type human papillomaviruses is an initial step for cervical carcinogenesis: association of clonal status and human papillomavirus infection with clinical outcome in cervical intraepithelial neoplasia. Lab Invest, 83, 1517-27.

Wright TC Jr, Cox JT, Massad LS, et al (2003). 2001 consensus guidelines for the management of women with cervical intraepithelial neoplasia. Am J Obstet Gynecol, 189, 295304. 